\title{
Ensayo Clínico de un Medidor Transcutáneo de Bilirrubina ${ }^{1}$
}

\author{
Dr Sergio Vaisman $W^{2}$ y Dra. Monica Ulinrichsen $\mathrm{M}^{2}$
}

\section{Clinical Trial with a Transcutaneous Bilirrubinometer}

\begin{abstract}
The clinical usefulness of a Transeutancous Bilirubinometer was studied in a group of 188 newbonns from the Salvador's Hospital. A total of 357 measurements were performed and the results were compated with the serum bilirubin concentrations obtained simultancously through a Moshidu Bilirubinometer. According to clinical chacateristics and treatment schedules, the newborns werc divided in 5 different groups. The results showed a significant correlation beween both methods in all of them, but in only 2 groups they were sufficiently high (r.79 and .91). At the present time routine clinical use' of the transitutaneous bilirubinoncter is not advisible according to this study.
\end{abstract}

En los últimos años hemos asistido a un avance tecnológico acelerado en todos los ámbitos de la medicina. En las Unidades Intensivas Neonatales, éste se ha enfocado en el diseño de instrutnentos que permitan la medición y control de distintos parámetros por métodos no invasivos. Se encuentran ya en uso rutinario el medidor transcutáneo de $\mathrm{pO}_{2}$, un método no invasivo de medición de la presión intracraneana, $y$ en forma experimental se está utilizando un medidor transcutáneo de $\mathrm{pCO}_{2}$ $y \mathrm{pH}$

El objetivo de nuestro estudio fue determinar la utilidad en la práctica clínica diaria de un método no invasivo de medición de ta bilirrubinemia utilizand o un medidor transcutáneo.

Material y Mètodo: El medidor transcutáneo de Bilirrubina es un espectrofotometro digital y manual, diseñado por las compañías Minolta y Air Shields. En un extremo tjene un dispositivo que se aplica sobre la piel del niño, generalmente en la región frontal. Ejerciendo una presión suave se produce la activación de un tubo de xenón el cual emite un haz de luz que viaja a través de filamentos de fibra óptica y llega a la piel del recién nacido, transiluminando el tejido subcutáneo. El haz de luz reflejado, vuelve, por otro sistema de fibras opticas, al módulo espectrofotométrico, donde es dividido en dos espectros por un espejo dicroico. Uno de los haces pasa a través de un filtro azul y el otro por un filtro verde. Con este

${ }^{1}$ Trabajo presentado en el 140 Congreso Chileno de Pediatria, Puerto Montt, Noviembre 1982

2 Departamento de Pediatría Hospital Calvo Mackenna, Fracultad de Medicina, División Orientu, Universidad de Chile. proceso se elimina la contribución de la hemoglobina en el resultado final. Por último, el estimulo luminoso es transformado en serial eléctrica que traducida en una cifra, se lee en la ventana del instrumento y corresponde a una escala arbitraria determinada por los fabricantes (Figura 1). La lectura obtenida con el aparato no corresponde directamente a la bilinubinemia, cuyo valor se descifrä desde un gráfico que establece la corretación entre un método de Laboratorio de medición y las lecturas del bilirrubinómetro transcutáneo (Figura 2).

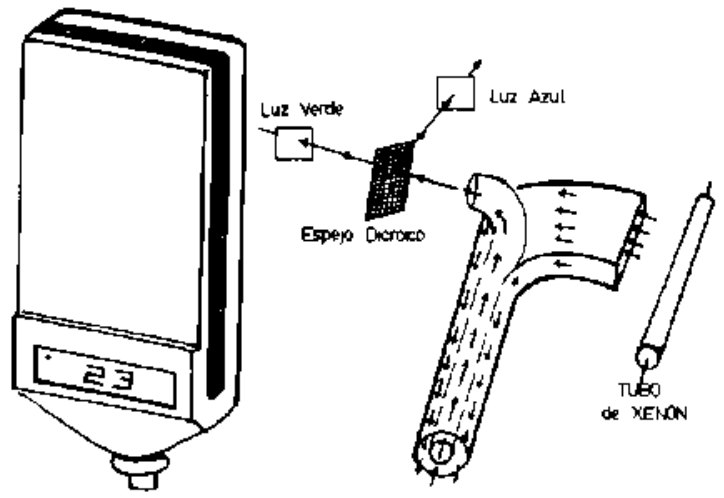

Figura 1.

Medidor Transcutáneo de Bilirrubina. Esquema de funcionamiento.

El estudio se realizó en un grupo de 188 recién nacidos de la Maternidad del Hospital Salvador que tenían indicación clínica para efectuar medición de bilirrubina sérica. Se efectuó un total de 357 mediciones y los recién nacidos se dividieron en 5 


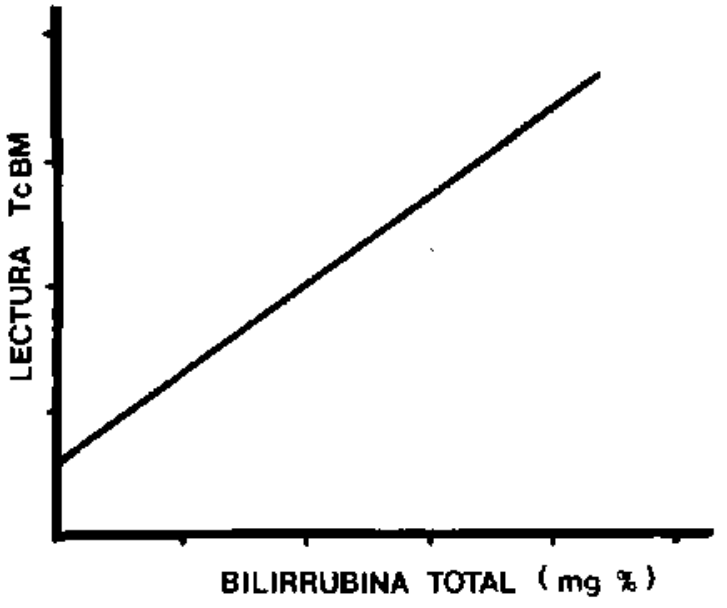

Figura 2.

Gráfico de corrclación entre lectura de bilirrabinómetro transızutánco y bitirtubinemia.

grupos: a) R.N. de Término en puerperio no sometidos a fototerapia $n=78$, b) R.N. deTérmino en fototerapia $n=77$, c) R.N. de Término en fototerapia post exanguíneo transfusión $\mathbf{n}=10$, d) R.N. Pretémino en fototerapian $=15(30-37$ semanas de edad gestacional) e) R.N. Ptetêrmino en puerperio no sometidos a fototerapia $n=8$ (36-37 semanas de edad gestacional). En cada uno de estos recién nacidos se realizaron mediciones en triplicado con el sistema transcutáneo durante cada uno de los dias en que permanecieron en el estudio. En forma simultánea se tomó muestra de sangre de talón para análisis de laboratorio de la bilitrubina sérica, utilizando el Bilirrubinómetro Moshida, el cual fue calibrado en forma periódica con solución estandar de bilirrubina marca Dade. Los resultados fueron sometidos a análisis estad ístico utilizando el coeficiente de correlación de Pearson.

Resultados: La Tabla J muestra los distintos grupos de recièn nacidos, el numero de lecturas efectuadas en cada uno de ellos, los promedios de lecturas con el Bilirrubinómetro Moshida y con el Bilirrubinómetro Transcutáneo. Se observa que en sólo dos grupos se obtiene correlaciones altas, pero a pesar de ello, estadísticamente la correlación es significativa en los cinco grupos estudiados $(\mathrm{p}<.05)$.

Tabla 1.

Correlación entre mediciones con el bilirubinómetro transcu táneo y bilirrubinemias de laboratorio en los 5 grupos de recién nacidos estudiados.

\begin{tabular}{|c|c|c|c|c|c|c|}
\hline & $\mathbf{n}$ & $\begin{array}{c}\text { No } \\
\text { Lesturas }\end{array}$ & $\begin{array}{c}\text { Bil. } \\
\text { Moshida }\end{array}$ & $\begin{array}{c}\text { Bi]. } \\
\text { I'tans:. }\end{array}$ & I & $\mathbf{p}$ \\
\hline ab R.R Técen as fotol. & 78 & 103 & 14.15 & 21.66 & 0.66 & S.ol \\
\hline b) R.N. Términn c/ fotol. & $7 ?$ & 174 & 14,02 & 21.01 & 0.46 & $<.01$ \\
\hline c) R.N J. diatod pos F.T. & 10 & 24 & 12.70 & 21.05 & 0.79 & $<\theta 1$ \\
\hline d) R N. Pr. J. : Fotot. & 15 & 47 & $1 \geq 38$ & 20.94 & $0.9 !$ & <.0] \\
\hline el.N. Pr. J. s/futol. & $B$ & 9 & 14.34 & 22.04 & 0.66 & $<.05$ \\
\hline
\end{tabular}

Las Figuras 3, 4, 5, 6 y 7 muestran las curvas de correlación en cada uno de los grupos estudiados. Se ve claramente que a medida que el valor de r disminuye, la dispersión en los valores de bilirnbina sérica aumenta para una misma lectura del Bilirrubinómetro Transcutáneo.

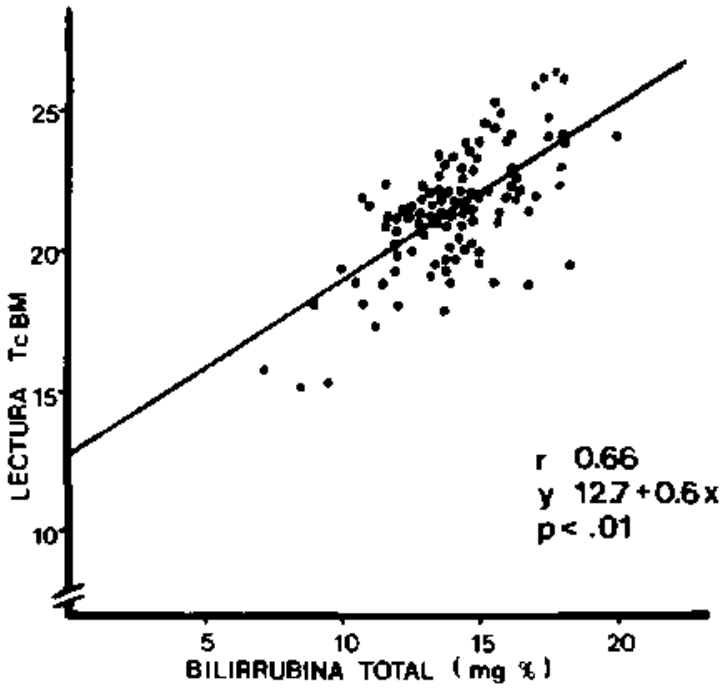

Figura 3.

Correlación entre bilirrubinómetro transcutáneo $y$ bilirubinemia total en 78 recién nacidos de término sin fototerapia.

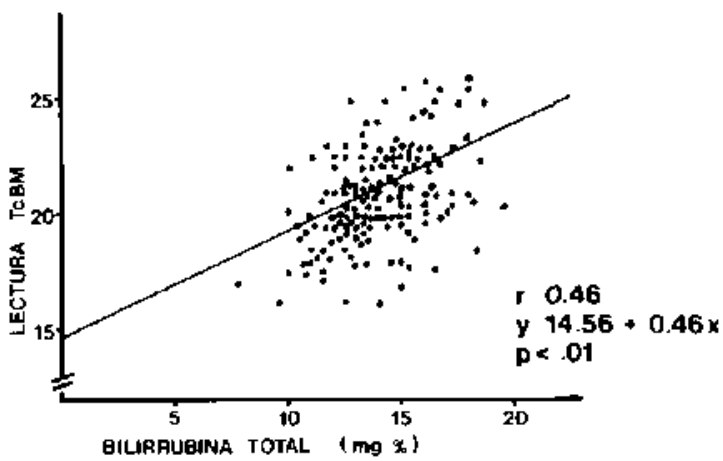

Figura 4.

Correlación ențre bilirrubinómetro transcu tánco y bilirrubinemia total en 77 recién nacidos de témino en fototerapia.

Discusión: Los resultados obtenidos en éste trabajo a pesar de mostrar una correlación estadisticamente significativa entre las lecturas del Bilirrubinómetro Transcutáneo y la medición efectuada en el laboratorio, sefialan, al menos en tres de los cinco grupos de recién nacidos analizados, valores de $r$ bajos que no permiten confiar ampliamente en su uso clínico. Nuestros resultados no concuerdan con otras publicaciones $\mathbf{1 \cdot 2 \cdot 3 \cdot 4}$ en que los valores 
de r en R.N. de Término sin fototerapia están entre .77 y .95 comparado con nuestro sesultado en un grupo similar que dio un $r$ de .66 . En R.N. de término con fototerapia se encuentra en la literatura valores de $r$ de .79 a $.86^{2 \cdot 3-4}$, comparado con nuestro valor de 46 .

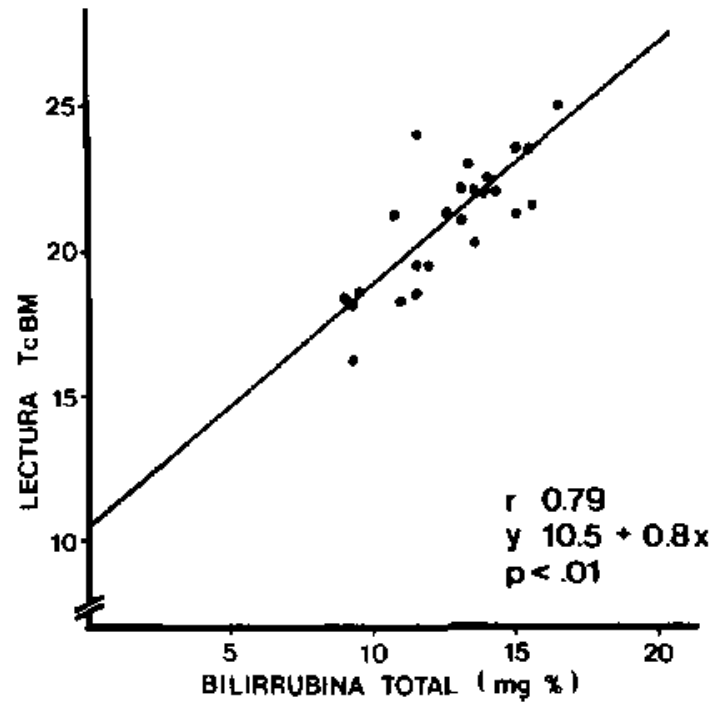

Figura 5.

Correlación entre bilirubinómetra transcutáneo y bilirubinemia to tal en 10 recién nacidos de término en fototerapia post exanguineo transtusión.

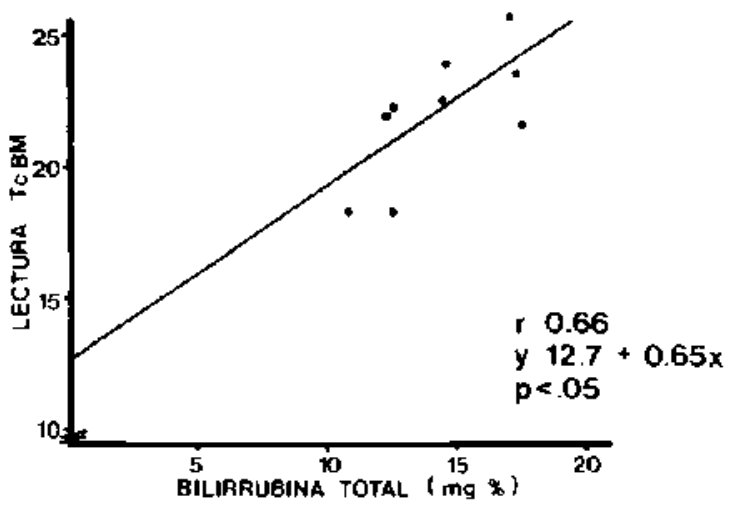

Figura 6.

Correlación en trc bilirrubinómetro transcutáneo y bilirrubinemira totak en 8 recién nacidos de pretérmino sin fototerapia.

Llama la atención que en los dos grupos en que encontramos una alta correlación, R.N.de Término con fototerapia post exanguineo transfusión $\mathrm{s}=$ .79 y R.N. Pretérmino con fototerapia $\mathrm{r}=.91$, las cifras de bilirrubinemia de laboratorio promedio son más bajas que en los otros grupos, siendo mayor la confiabilidad del instrumento en los niveles más bajos de bilirrubinemia. Esto es ava-
Lado por los resultados de Hegyi y cols. ${ }^{2}$ que muestra una correlación significativamente más alta en el grupo de nir̃os con bilirrubina sérica bajo $10 \mathrm{mg} \%$ comparado con un grupo con niveles de bilirrubina sobre estas cifras. Esta podría ser la explicación de la aparente menor correlación encontrada por nosotros comparada con otras publicaciones que, ademas de considerar un número pequeño de mediciones. incorporarian recién nacidos con niveles más bajos de bilirrubinemia.

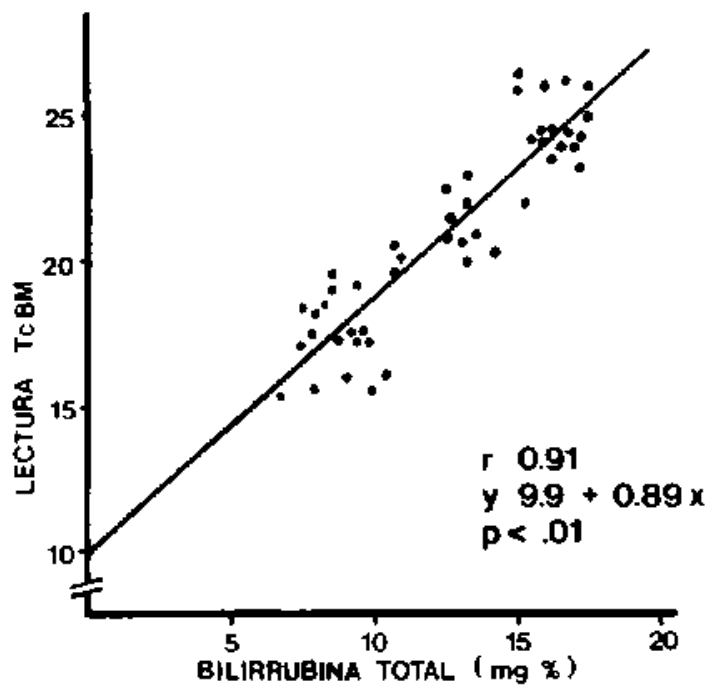

Figura 7.

Correlación entre bilirrubinómetro transcutáneo y bilirrubinemia total en 15 recién nacidos de pretermino en fototerapia.

En el grupo de R.N. de Término sometidos a fototerapia post exanguíneo transfusión obtuvimos una correlación aceptable. Esto discrepa con la impresión inicial de Yamanouchi y cols. ${ }^{1}$, pero él analizó un solo niño sometido a este procedimiento al que controló durante 7 días. En nuestro grupo, que incluye 10 niños sometidos a 24 lecturas con el Bilirrubinómetro Transcutáneo, la correlación es aceptable $(r=79)$ y corresponde a un promedio de bilirrubina sérica más baja que los otros grupos (12.7 mg \%).

No analizamos el tipo de población incorporada a nuestro estudio, por tratarse, en general, de una población bastante homogénea. Diversos autores han encontrado diferencias raciales según se trate de raza blanca, negra u oriental, pero los resultados publicados hasta ahora no son concluyentes $2-3-4-5-6$

Estudios previos han demostrado la mayor confuabilidad de las lecturas en la frente ${ }^{1}$, zona que elegimos para el estudio. Por el efecto de la fototerapia en decolorar la piel, usamos un parche de 3 x $3 \mathrm{~cm}$. de material aislante de la luz, y que fue 
colocado en la frente del recién nacido en que se indicaba la fototerapia. Sin embargo, Sun y cols. ${ }^{7}$, no encontraron diferencias, al efectuar mediciones simultáneas en la frente de niños sometidos a fototerapia entre una zona cubierta con parche y otra contigua expuesta a la luz por lo que parecería innecesario st uso.

Los valores de nuestras lecturas en triplicudo fueron analizados mediante el coeficiente de variación que dio un valor promedio de $2.47 \%$, demostrando la confiabilidad del instrumento en la reproducibilidad de sus mediciones.

Estadísticamente la correlación encontrada en los distintos grupos es significativa, pero cuando se necesita desde el punto de vista clínico un instrumento confiable para medir la bilirrubina, la correlación tiene que ser sobre un $90 \%$. No nos parece útil la conclusión de $\mathrm{A}$. Brown y cols. ${ }^{3}$, en que con lecturas del instrumento sobre ciertos valores sería necesario efectuar una determinación de bilirrubina sérica, puesto que el problema se le plantea al clínico justamente con los valores altos de bilimubinemia. Con cifras bajas, es decir, en recién nacidos con ictericias moderadas, la posibilidad de equivocarse con la evaluación clínica es menor y es menos trascendente para el paciente por lo que se necesita una mayor confiabilidad del instrumento en las ictericias intensas.

Es extraordinariamente ventajoso contar con un método no invasivo de determinación de los niveles de ictericia, lo que permite mejor eficiencia clínica y menor riesgo para el recién nacido, con un procedimiento rápido, inocuo y de un costo relativamente bajo, si se considera el costo de las técnicas actualmente en uso. Creemos que en el momento actual se requiere más ensayos clínicos con un número mayor de pacientes estudiados para obtener conclusiones definitivas en relación al medidor transcutáneo de Bilirrubina.

Nuestros resultados no permiten aconsejar su uso clínico rutinario y en esto concordamos con Hannemann y cols. ${ }^{4}$ y con Goldman y cols. ${ }^{8}$. Sin embargo, creemos que el concepto tecnológico involucrado es interesante $y$ es posible que con algu- nas modificaciones introducidas por el fabricante, se transforme en un futuro próximo en una técnica de uso habitual.

Resumen: Se estudia la confiabilidad clínica de un Bilirrubinòmetro Transcutáneo en un grupo de 188 Recién Nacidos de la Maternidad del Hospital Salvador con un total de 357 mediciones, comparándolo con las mediciones de bilirrubina sérica efectuadas en ur Bilirrubinómetro estandar. El total de niños estudiados se divide en 5 . grupos distintos de acuerdo a sus caracteristicas clínicas y tratamientos efectuados, encontrándose una correlación estadísticamente significativa en todos ellos, pero sólo en 2 grupos con valores de $\mathrm{r}$ elevados (.79 y 91). No se aconseja por ahora su uso clínico rutinario y se comentan otras publicaciones extranjeras que utilizan el mismo instrumento.

\section{REFERENCIAS}

1 Yamanouchi L., Yamauchi Y. and Igorashi l.: Transcutaneous Bilirubinometry: preliminacy studies of non invasive transcutaneous bilirubin meter in the Okayama National Hospital, Pediatrics 65: 195; 1980.

${ }^{2}$ Hegyi I, Hiatt I.M. and Indyk L.: Transcutaneous Bilirubinometry. Correlations in term infants. J. Pediat. 98: 454: 1981 .

${ }^{3}$ Brown A.K., Kim M.H. Nuchpuckdee P. and Boyle G.: Transcutaneous Bilirubinometry in infants: Influence of race and phototherapy. Ped. Res. 15: 653:1981.

4 Hannemann R.E., Schreiner R.L., Dewitt D.P., Norris $S . A$, and Glick M.R.: Evatuation of the Minolta Bilj. rubin Meter as a screening device in white and black infants. Pediatries 69: 107; 1982.

5 Engel R.R., Henis B.B., Engel R.E. and Bandt C.: Effect of race and other variables on transcutaneous bilirubj. nometry. Ped. Res. 15: 531; 1981.

6 Vangyanichyakom $K$., Sun $S$, Abubaker A. and Glisto B.: Transcutaneous Bilirubinometry in black and hispanic infants Ped. Res. 15; 685; 1981

'Sun S., Vangyanickyakom K., Nutakul L., Ranee $S$. and Glisfa B.: Transcutancous Bilirubinometry in black pretem intants on covered and exposed skin areas. Ped. Ros. 15: 684; 1981.

${ }^{8}$ Goldiman S.L., Peñalver A. and Peñaranda R.: Iaudice meter: evaluation of new guidelines. J. Pediat, 101: $253 ; 1982$. 